\title{
Improvement of an Atomic Clock using Squeezed Vacuum
}

\author{
I. Kruse, ${ }^{1}$ K. Lange, ${ }^{1}$ J. Peise, ${ }^{1}$ B. Lücke, ${ }^{1}$ L. Pezzè,${ }^{2}$ J. Arlt, ${ }^{3}$ W. Ertmer, ${ }^{1}$ C. Lisdat, ${ }^{4}$ L. Santos, ${ }^{5}$ A. Smerzi,${ }^{2}$ and C. Klempt ${ }^{1}$ \\ ${ }^{1}$ Institut für Quantenoptik, Leibniz Universität Hannover, Welfengarten 1, D-30167 Hannover, Germany \\ ${ }^{2}$ QSTAR, INO-CNR and LENS, Largo Enrico Fermi 2, I-50125 Firenze, Italy \\ ${ }^{3}$ Institut for Fysik og Astronomi, Aarhus Universitet, Ny Munkegade 120, DK-8000 Århus C, Denmark \\ ${ }^{4}$ Physikalisch-Technische Bundesanstalt, Bundesallee 100, D-38116 Braunschweig, Germany \\ ${ }^{5}$ Institut für Theoretische Physik, Leibniz. Universität Hannover, Appelstraße 2, D-30167 Hannover, Germany
}

(Received 25 May 2016; published 28 September 2016)

\begin{abstract}
Since the pioneering work of Ramsey, atom interferometers are employed for precision metrology, in particular to measure time and to realize the second. In a classical interferometer, an ensemble of atoms is prepared in one of the two input states, whereas the second one is left empty. In this case, the vacuum noise restricts the precision of the interferometer to the standard quantum limit (SQL). Here, we propose and experimentally demonstrate a novel clock configuration that surpasses the SQL by squeezing the vacuum in the empty input state. We create a squeezed vacuum state containing an average of 0.75 atoms to improve the clock sensitivity of 10000 atoms by $2.05_{-0.37}^{+0.34} \mathrm{~dB}$. The SQL poses a significant limitation for today's microwave fountain clocks, which serve as the main time reference. We evaluate the major technical limitations and challenges for devising a next generation of fountain clocks based on atomic squeezed vacuum.
\end{abstract}

DOI: 10.1103/PhysRevLett.117.143004

Precision measurements allow us to probe the boundaries of our understanding of physics. Prominent recent examples include the discovery of gravitational waves with optical interferometers [1] and the improving bounds on the drift of fundamental constants with atomic interferometers $[2,3]$. The current generation of atomic and optical interferometers is, however, fundamentally limited by vacuum noise, the so-called standard quantum limit (SQL). Squeezing the vacuum entering one port of an optical gravitational wave detector was proposed in the 1980s [4] to surpass the SQL when measuring the length difference between two optical paths. Can squeezed vacuum be useful to improve the measurement of time? Up to now, the concept of vacuum squeezing has not been transferred to atomic clocks or atom interferometry in general.

In this Letter, we design and implement an atom interferometer in clock configuration which exploits the atomic squeezed vacuum. The clock is operated by combining $N=10^{4}$ atoms in one input state with a quadraturesqueezed vacuum with an average of 0.75 atoms in the second input state. The squeezed vacuum is generated by spin-changing collisions in a Bose-Einstein condensate of neutral ${ }^{87} \mathrm{Rb}$ atoms - in direct analogy to optical parametric down-conversion [5-7]. In contrast to existing methods [8-17] to increase the sensitivity of atomic clocks beyond the SQL in large ensembles, our concept disentangles the challenge of increasing the number of atoms (in the main input state) from the creation of squeezing (in the vacuum state). In particular, the vacuum state remains weakly populated during its preparation, making it immune to losses. These central advantages are also exploited in squeezed-vacuum optical interferometers for the detection of gravitational waves, as demonstrated in GEO600 [18] and LIGO [19], where coherent states of $>10 \mathrm{~W}$ are combined with a low-power squeezed vacuum state to achieve sub-SQL measurement uncertainty.

Our atomic clock consists of a four-mode linear Ramsey interferometer when described in terms of the Zeeman states $| \pm 1\rangle=\left|F=1, m_{F}= \pm 1\right\rangle, \quad|0\rangle=\left|F=1, m_{F}=0\right\rangle$, and $|e\rangle=\left|F=2, m_{F}=0\right\rangle$ (see Fig. 1). It can be reduced to a standard two-level Ramsey sequence in terms of the magnetically insensitive clock states $|g\rangle=(|+1\rangle+|-1\rangle) /$ $\sqrt{2}$ and $|e\rangle$ (see Fig. 1 and Ref. [20]). The atoms are prepared in a balanced superposition of $|0\rangle$ and $| \pm 1\rangle$ by a radio-frequency (rf) $\pi / 2$ pulse, which couples the states $|0\rangle$ and $|g\rangle$ [pulse I in Fig. 1(a)]. A subsequent microwave (mw) $\pi$ pulse transfers the atoms from $|0\rangle$ to $|e\rangle$ (II). In this superposition state, the atoms sense the evolution of time by acquiring a phase shift $\theta=2 \pi \tau \delta$, which depends on the detuning $\delta$ of the employed microwave oscillator and the phase evolution time $\tau=\tau_{R}+\tau_{\mathrm{mw}}$, where $\tau_{R}$ is the Ramsey interrogation time and $\tau_{\mathrm{mw}}$ is the microwave pulse duration. A second mw $\pi$ pulse transfers atoms back in $|0\rangle$ (III). Finally, a second rf pulse (IV) closes the interferometer. The phase is estimated from the fraction $f=N_{g} /$ $N \approx N_{g} /\left(N_{g}+N_{0}\right) \approx\left(N_{+1}+N_{-1}\right) / N$ of atoms in the output state $|g\rangle$, which can be obtained directly from an absorption image of all three Zeeman components. These Zeeman components are spatially separated by a magnetic gradient during free fall. Subsequently, absorption imaging is performed on the closed transition $F=2 \rightarrow F^{\prime}=3$ on the $\mathrm{Rb} D 2$ line with a resonant laser beam derived from an 
(a)

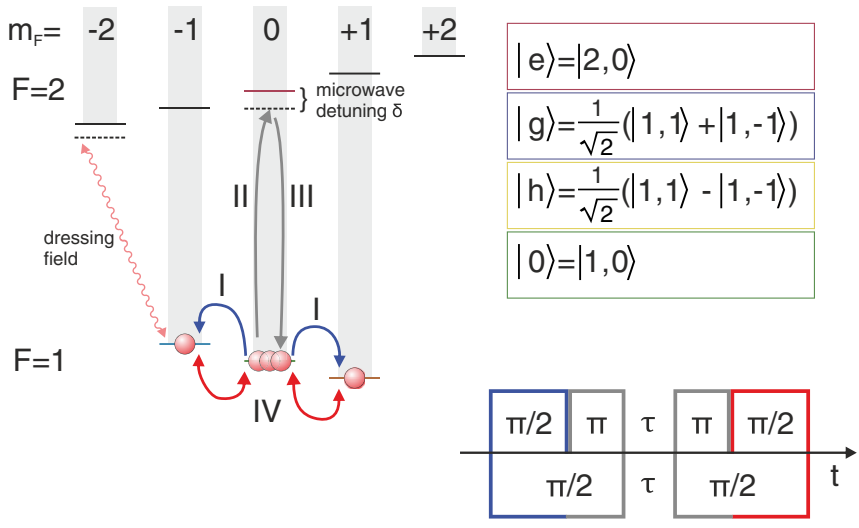

(b)

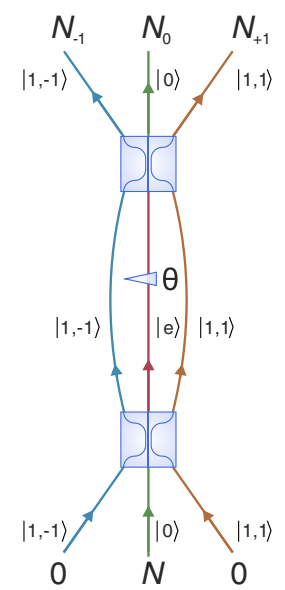

(c)

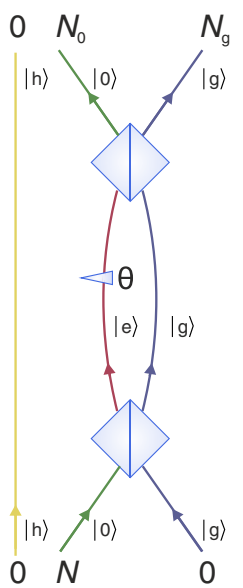

FIG. 1. The three-mode interferometer. (a) Hyperfine ground states of ${ }^{87} \mathrm{Rb}$. (I) A Bose-Einstein condensate in the state $|0\rangle=$ $\left|F=1, m_{F}=0\right\rangle$ is coupled to the states $|1, \pm 1\rangle$ by a resonant radio-frequency pulse. The second-order Zeeman shift is compensated by a detuned microwave dressing to the state $|2,-2\rangle$. (II) A microwave pulse with detuning $\delta$ couples the states $|0\rangle=|1,0\rangle$ and $|e\rangle=|2,0\rangle$. The radio-frequency and microwave pulses form an effective $\pi / 2$ pulse between $|1, \pm 1\rangle$ and $|e\rangle$. (III) After a Ramsey evolution time $\tau_{\mathrm{R}}$, a second detuned microwave pulse is applied. (IV) A final radio-frequency pulse coupling $|0\rangle$ and $|1, \pm 1\rangle$ closes the interferometer. (b) The interferometer corresponds to two three-mode beam splitters and a phase shift in between. The total number of atoms $N$ enters the central state $|0\rangle$. A measurement of all three components $N_{-1,0,1}$ after the interferometer allows for an estimation of the phase shift $\theta$. (c) The interferometer can be simplified by introducing the symmetric state $|g\rangle$ and the antisymmetric state $|h\rangle$. The three-mode beam splitters only couple to the symmetric state, thus yielding an effective two-mode interferometer with an unchanged antisymmetric state.

external-cavity diode laser. Atoms in $F=1$ are pumped to the cycling transition by an independent repumping laser beam on the transition $F=1 \rightarrow F^{\prime}=2$. The detection noise of 16 atoms ( $7 \mathrm{~dB}$ below the projection noise level) is dominated by the photoelectron shot noise on the CCD camera and does not limit the experimental results. Figure 2(a) presents the Ramsey fringes for the classical case, when the hyperfine level $|g\rangle$ is initially empty. The average fraction $\bar{f}$ is shown as a function of the microwave detuning $\delta$ for two Ramsey times $\tau_{R}$ and is well reproduced by a single-atom model [20]. The slightly reduced contrast for larger $\tau_{R}$ stems mainly from the influence of a small radio-frequency detuning as well as magnetic field noise. In addition, the model accounts for the fixed pulse length $\tau_{\mathrm{mw}}$ for all detunings, leading to a small amount of atoms remaining in the $F=2$ manifold $\left(<2.5 \%\right.$ for $\tau_{R}=250$ and $<8 \%$ for $\tau_{R}=1000 \mu$ s for up to $2 \mathrm{kHz}$ detuning). In the limit of weak magnetic field gradients, these atoms experience the same spatial separation as the atoms in $F=1$ and thus cannot be discriminated by our absorption imaging. In the following, the pulse length is adjusted such that no atoms remain in the $F=2$ manifold. For small $\tau_{R}$ the data are in good agreement with the noiseless prediction $\bar{f}=\sin ^{2} \pi \delta \tau$.

The clock performance is evaluated for a vanishing time $\tau_{R}=0$ between the two detuned microwave pulses (II and III) to minimize technical noise. For a microwave pulse length of $2 \tau_{\mathrm{mw}}=90.4 \mu \mathrm{s}$ and a detuning of $\delta=-5.5 \mathrm{kHz}$ we reach the midfringe position $\theta=2 \pi \tau_{\mathrm{mw}} \delta=\pi / 2$, where the slope $\partial \bar{f} / \partial \theta$ has its maximum value $1 / 2$. Here, $\tau_{\mathrm{mw}}$ is chosen such that all atoms return to the state $|0\rangle$. On midfringe position, the full interferometer sequence can be described as a single, symmetric beam splitter between $|0\rangle$ and $|g\rangle$. Because of the large number of atoms in the state $|0\rangle$, which act as a local oscillator with a defined phase $\varphi$ in the quantum optics sense, the interferometer sequence presents a standard homodyne measurement of the quadratures in state $|g\rangle$. Therefore, the fluctuations of the interferometer output reflect the quadrature fluctuations: $(\Delta f)^{2}=(\Delta X)^{2} /(2 N)$ [20]. Here, $X=1 / \sqrt{2}\left(e^{-i \varphi} g+\right.$ $\left.e^{i \varphi} g^{\dagger}\right)$ and $P=1 / i \sqrt{2}\left(e^{-i \varphi} g-e^{i \varphi} g^{\dagger}\right)$ are quadrature operators of the symmetric state $|g\rangle$, defined in terms of the creation and annihilation operators $g^{\dagger}$ and $g$, respectively, and $\varphi$ is the local oscillator phase. With an initially empty state $|g\rangle$, the quadrature fluctuation is $(\Delta X)^{2}=1 / 2$. This limits the ideal phase estimation uncertainty to $(\Delta \theta)^{2}=$ $(\Delta f)^{2} /(\partial \bar{f} / \partial \theta)^{2}=1 / N$. In our experiments, we record a value of $(\Delta f)^{2}=1.48 / N$, which is $1.69 \mathrm{~dB}$ above the vacuum limit due to technical noise mainly caused by magnetic field fluctuations [20].

The sensitivity of our interferometer can surpass the SQL when quadrature fluctuations are squeezed below the vacuum limit, $(\Delta X)^{2}<1 / 2$. We create a squeezed vacuum state by initiating spin dynamics in the Bose-Einstein condensate prior to the interferometer sequence. Spin dynamics can be precisely controlled by switching the 

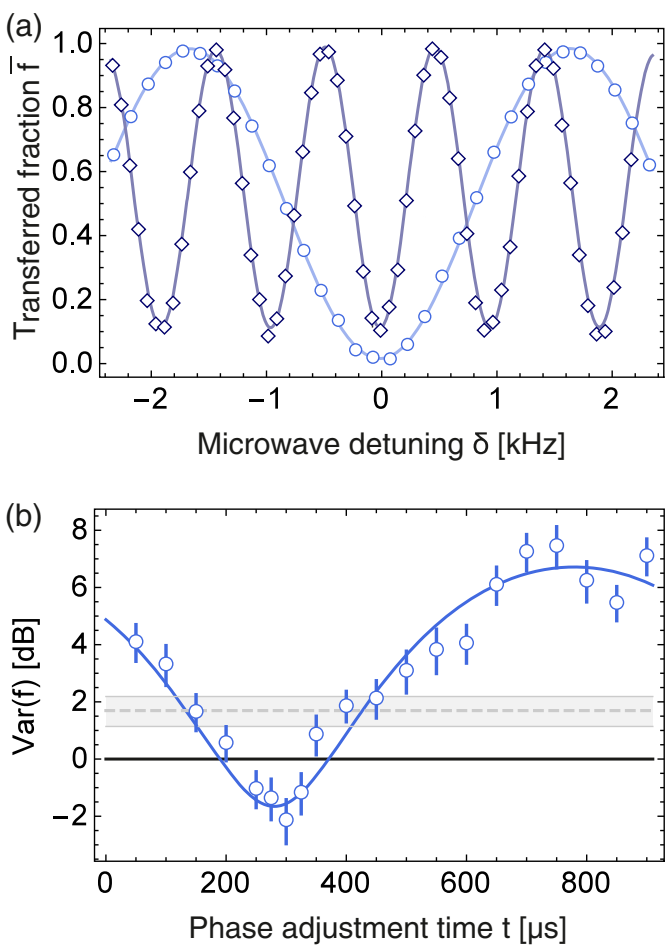

FIG. 2. Output of the interferometer. (a) The microwave detuning $\delta$ is varied for different Ramsey times $\tau_{R}=250$ (open circles) and $\tau_{R}=1000 \mu \mathrm{s}$ (open diamonds). The phase shift, set by the microwave detuning ( $x$ axis) and the evolution time $\tau=\tau_{R}+\tau_{\mathrm{mw}}$, results in the Ramsey fringes in the transferred fraction. The solid lines (dark and light) represent the results of our model. (b) The phase adjustment time $t$ before the interferometer sequence is varied and the corresponding variance of the transferred fraction is recorded with respect to projection noise (open circles). The data are well reproduced by a sinusoidal fit (light blue line) and reaches clearly below projection noise (solid black line). Result of the classical interferometer (shaded dashed area). The error bars and shaded areas represent the statistical uncertainty of 100 repetitions.

microwave dressing of the state $|-1\rangle$ to the resonance condition $[7,29]$. In direct analogy to optical parametric down-conversion, spin dynamics creates pairs of atoms according to the two-mode squeezing Hamiltonian $H=$ $\hbar \Omega\left(a_{+1}^{\dagger} a_{-1}^{\dagger}+a_{+1} a_{-1}\right)[5,20,30]$, where $a_{ \pm 1}^{\dagger}$ and $a_{ \pm 1}$ are the creation and annihilation operators for atoms in $| \pm 1\rangle$, and $\Omega=2 \pi \times 3.9 \mathrm{~s}^{-1}$ is the spin dynamics rate. Using the operators $g=\left(a_{+1}+a_{-1}\right) / \sqrt{2}$ and $h=\left(a_{+1}-a_{-1}\right) / \sqrt{2}$, this Hamiltonian simplifies to $H=H_{g}-H_{h}$, with $H_{g}=$ $\hbar \Omega / 2\left(g^{\dagger} g^{\dagger}+g g\right)$ and the analogous definition for $H_{h}$. Spin dynamics $e^{-i H t / \hbar}=e^{-i r\left(g^{\dagger} g^{\dagger}+g g\right) / 2} \otimes e^{i r\left(h^{\dagger} h^{\dagger}+h h\right) / 2}$, equivalent to a two-axis countertwisting dynamics $[31,32]$ (see Ref. [20]), can thus be written in terms of the product of usual single-mode quadrature-squeezing operators [33], where $r=\Omega t$. We notice that $H_{g}$ and $H_{h}$ rewrite as two-axis countertwisting Hamiltonians $[31,32]$ for opportune spin operators; see Ref. [20]. We apply spin dynamics for $32 \mathrm{~ms}$, which creates a mean number $\sinh ^{2} r=0.75$ of atoms in each of the two states. This number is extremely small compared to the total of $N \approx 10^{4}$ atoms, such that the influence of the antisymmetric state to the interferometer signal is negligible and we can approximate $f=$ $\left(N_{+1}+N_{-1}\right) / N=\left(N_{g}+N_{h}\right) / N \approx N_{g} / N$. Even though the symmetric state $|g\rangle$ is only weakly populated, it has a strong influence on the interferometric sensitivity. The squeezing allows for reduced quadrature fluctuations of $(\Delta X)^{2}=(1 / 2) e^{-2 r}<(1 / 2)$ for $r>0$ at an optimal local oscillator phase $\varphi=\pi / 4$. Experimentally, the phase $\varphi$ is adjusted by applying a controlled energy shift with the microwave dressing field for a variable duration $t$ prior to the interferometer sequence.

Figure 2(b) shows the variance of the population imbalance $(\Delta f)^{2}$ as a function of the adjusted phase relation. At an optimum value of $t=300 \mu \mathrm{s}$, a minimal variance of $-2.12_{-0.83}^{+0.70} \mathrm{~dB}$ below projection noise is reached. The variances are obtained from a total number of 100 repetitions of the experiment per phase adjustment time $t$. All error bars indicate 1 standard deviation of the statistical uncertainty and are obtained by an unbiased estimation (see Supplements of Ref. [7]). Figure 3(a) shows the fraction $\bar{f}$ as a function of the detuning in the vicinity of the midfringe position, as obtained from 390 experimental realizations for each microwave detuning $\delta$. The slope is proportional to the contrast of the interferometer and depends on the coherence properties of the input state. A fit (blue solid line) yields a value of $0.48 \mathrm{rad}^{-1}$, which is close to the optimal value of $0.5 \mathrm{rad}^{-1}$. The variances of the population imbalance and the fitted slope yield the phase estimation uncertainty $(\Delta \theta)^{2}=(\Delta f)^{2} /(\partial \bar{f} / \partial \theta)^{2}$ displayed in Fig. 3(b). At a detuning of $-5.9 \mathrm{kHz},(\Delta \theta)^{2}$ reaches a minimum value $-1.56_{-0.45}^{+0.41} \mathrm{~dB}$ below the SQL. The two-sample variance, which rejects long term technical drifts and is therefore better suited to estimate the fundamental noise, reaches $-2.05_{-0.37}^{+0.34} \mathrm{~dB}$ below the SQL.

The interferometric measurements also allow for a reconstruction of the squeezed vacuum state in mode $|g\rangle$. The Wigner function in $X-P$ space after the optimal phase adjustment time of $300 \mu \mathrm{s}$ is obtained from an inverse Radon transformation [see Fig. 4(a) and Ref. [20]]. Its profile is very close to the expected Gaussian distribution, and is characterized by the squeezed and antisqueezed widths along the $X$ and $P$ directions, respectively. While this single-mode picture successfully describes the physics of our experiments, it can equivalently be described by spin squeezing of the usual two-mode pseudospin operators, as visualized on the multiparticle Bloch sphere in Fig. 4(b). It is worth noting, that these collective pseudospin operators are identical to the SU(2) subspaces exhibiting spin-nematic squeezing reported in Ref. [35]. Furthermore, the created squeezed vacuum state can also be employed for phase sensing in a nonlinear interferometer scheme of the $\mathrm{SU}(1,1)$ type $[36,37]$. 


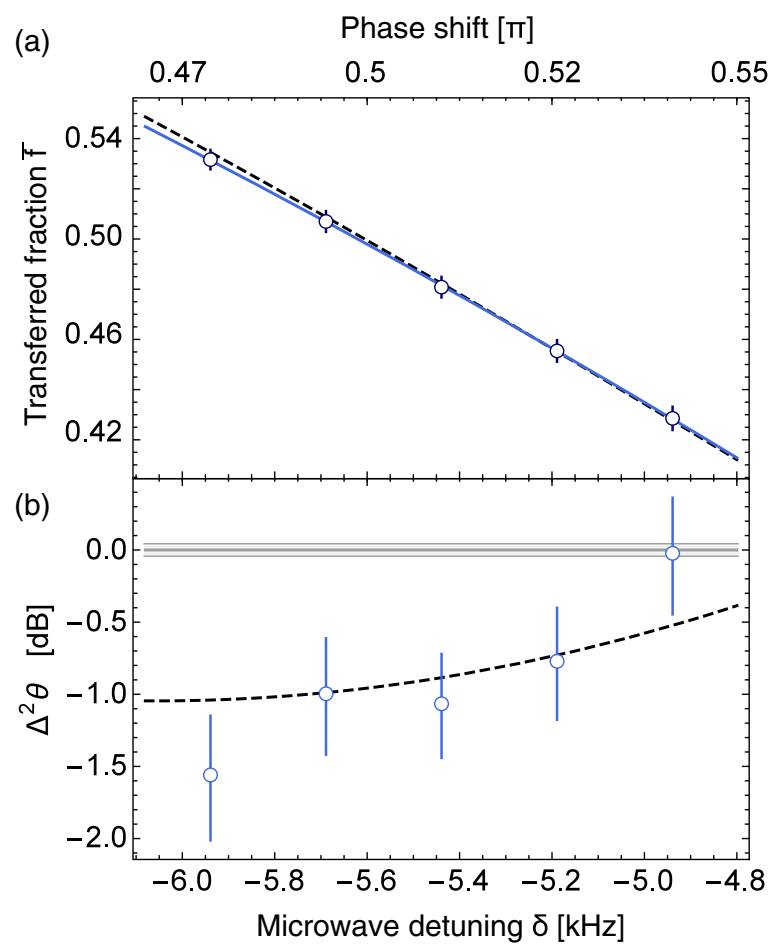

FIG. 3. Phase estimation uncertainty. (a) Mean values of the transferred fraction $\bar{f}$ (black open circles) for five different microwave detunings (bottom $x$ axis) and corresponding phase shifts (top $x$ axis) close to the midfringe position. The slope of the linear fit (blue solid line) is close to the optimum, as represented by our model (black dashed line). The error bars represent the (sub-projection noise) statistical uncertainty of 390 repetitions. (b) The phase estimation uncertainties $\Delta^{2} \theta$ normalized to the SQL (blue open circles) reach well below the SQL (black dashed line). The shaded area represents an upper bound of the uncertainty of the atom number calibration of $1 \%$ [34]. The small detuning dependency of the recorded sensitivity is reproduced by our single-atom model including magnetic field noise (black dotted line). The best phase estimation uncertainty of $-1.56_{-0.45}^{+0.41} \mathrm{~dB}$ below the SQL is reached at a detuning of $-5.9 \mathrm{kHz}$. Error bars are the statistical uncertainty (1 standard deviation) of 390 repetitions.

In summary, our experiments present the first proof-ofprinciple implementation of squeezed vacuum in an atomic microwave clock. Microwave fountain clocks, providing the realization of the SI second, are currently limited by the SQL [38-41]. In combination with the recently developed sources of Bose-Einstein condensed atoms with small densities $[42,43]$ and high repetition rates [44], our results pave the way for the development of a new generation of atomic microwave clocks operating beyond the SQL [20]. Our method is particularly robust during state preparation. In contrast to existing proposals, it avoids the generation of entangled states with a symmetric population of the two hyperfine levels, which is plagued by two-body losses in the excited hyperfine state. The limitations of our method for sub-SQL interferometry have not been reached yet: (a)

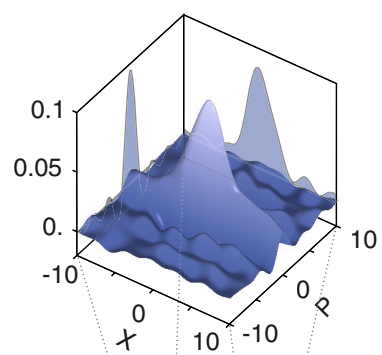

(b)

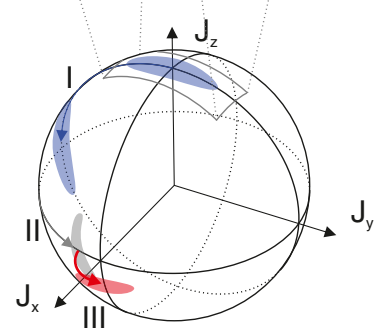

FIG. 4. Reconstructed Wigner function and Bloch sphere representation. (a) The data presented in Fig. 2(b) are used to reconstruct the two-dimensional Wigner function in the $X-P$ space. Here, the Wigner function of the state after the optimal phase adjustment time of $t=300 \mu \mathrm{s}$ is shown. (b) The interferometer is illustrated on the multiparticle Bloch sphere for the states $|g\rangle$ and $|e\rangle$, in terms of the pseudospin operators $J_{x}=(1 / 2)\left(e^{\dagger} g+g^{\dagger} e\right), J_{y}=1 /(2 i)\left(e^{\dagger} g-g^{\dagger} e\right), J_{z}=(1 / 2)\left(e^{\dagger} e-\right.$ $\left.g^{\dagger} g\right)$. The employed squeezed vacuum corresponds to an elliptical uncertainty disk with variable orientation angle depending on the phase adjustment time $t$. An optimal orientation angle, as shown, allows for a measurement of the transferred fraction with a sub-projection-noise uncertainty. The two radio-frequency pulses generate rotations around the $J_{x}$ axis (I/III). The phase shift corresponds to a rotation around the $J_{z}$ axis (II).

besides overcoming technical restrictions, it has been shown [45] that an optimized version of the present interferometric scheme can reach the ultimate Heisenberg limit of phase sensitivity $\Delta \theta=1 / N$.

We thank T. Opatrny for comments. We acknowledge support from the Centre for Quantum Engineering and Space-Time Research (QUEST) and from the Deutsche Forschungsgemeinschaft through CRC 1227 (DQ-mat), project A02, Research Training Group 1729, and the EMRP project QESOCAS. The EMRP is jointly funded by the EMRP participating countries within EURAMET and the European Union. J. A. acknowledges support by the Lundbeck Foundation.

[1] LIGO Scientific Collaboration and Virgo Collaboration, Phys. Rev. Lett. 116, 061102 (2016).

[2] N. Huntemann, B. Lipphardt, C. Tamm, V. Gerginov, S. Weyers, and E. Peik, Phys. Rev. Lett. 113, 210802 (2014).

[3] R. M. Godun, P. B. R. Nisbet-Jones, J. M. Jones, S. A. King, L. A. M. Johnson, H. S. Margolis, K. Szymaniec, S. N. Lea, K. Bongs, and P. Gill, Phys. Rev. Lett. 113, 210801 (2014). 
[4] C. M. Caves, Phys. Rev. D 23, 1693 (1981).

[5] C. Klempt, O. Topic, G. Gebreyesus, M. Scherer, T. Henninger, P. Hyllus, W. Ertmer, L. Santos, and J. J. Arlt, Phys. Rev. Lett. 104, 195303 (2010).

[6] M. Scherer, B. Lücke, G. Gebreyesus, O. Topic, F. Deuretzbacher, W. Ertmer, L. Santos, J. J. Arlt, and C. Klempt, Phys. Rev. Lett. 105, 135302 (2010).

[7] B. Lücke, J. Peise, G. Vitagliano, J. Arlt, L. Santos, G. Tóth, and C. Klempt, Phys. Rev. Lett. 112, 155304 (2014).

[8] J. Appel, P. J. Windpassinger, D. Oblak, U. B. Hoff, N. Kærgaard, and E. S. Polzik, Proc. Natl. Acad. Sci. U.S.A. 106, 10960 (2009).

[9] W. Wasilewski, K. Jensen, H. Krauter, J. J. Renema, M. V. Balabas, and E. S. Polzik, Phys. Rev. Lett. 104, 133601 (2010).

[10] M. H. Schleier-Smith, I. D. Leroux, and V. Vuletić, Phys. Rev. Lett. 104, 073604 (2010).

[11] J. G. Bohnet, K. C. Cox, M. A. Norcia, J. M. Weiner, Z. Chen, and J. K. Thompson, Nat. Photonics 8, 731 (2014).

[12] N. Behbood, F. Martin Ciurana, G. Colangelo, M. Napolitano, G. Tóth, R. J. Sewell, and M. W. Mitchell, Phys. Rev. Lett. 113, 093601 (2014).

[13] O. Hosten, N. J. Engelsen, R. Krishnakumar, and M. A. Kasevich, Nature (London) 529, 505 (2016).

[14] C. Gross, T. Zibold, E. Nicklas, J. Estève, and M. K. Oberthaler, Nature (London) 464, 1165 (2010).

[15] M. Riedel, P. Böhi, Y. Li, T. Hänsch, A. Sinatra, and P. Treutlein, Nature (London) 464, 1170 (2010).

[16] H. Strobel, W. Muessel, D. Linnemann, T. Zibold, D. B. Hume, L. Pezzè, A. Smerzi, and M. K. Oberthaler, Science 345, 424 (2014).

[17] B. Lücke, M. Scherer, J. Kruse, L. Pezzé, F. Deuretzbacher, P. Hyllus, O. Topic, J. Peise, W. Ertmer, J. Arlt, L. Santos, A. Smerzi, and C. Klempt, Science 334, 773 (2011).

[18] LIGO Scientific Collaboration, Nat. Phys. 7, 962 (2011).

[19] J. Aasi, J. Abadie, B. P. Abbott, R. Abbott, T. D. Abbott, M. R. Abernathy, C. Adams, T. Adams, P. Addesso, R. X. Adhikari et al., Nat. Photonics 7, 613 (2013).

[20] See Supplemental Material at http://link.aps.org/ supplemental/10.1103/PhysRevLett.117.143004 for information on experimental sequence, theoretical model, and influence of noise, which includes Refs. [21-28].

[21] Y.-J. Lin, A. R. Perry, R. L. Compton, I. B. Spielman, and J. V. Porto, Phys. Rev. A 79, 063631 (2009).

[22] R. J. Lewis-Swan and K. V. Kheruntsyan, Phys. Rev. A 87, 063635 (2013).

[23] S. P. Nolan, J. Sabbatini, M. W. J. Bromley, M. J. Davis, and S. A. Haine, Phys. Rev. A 93, 023616 (2016).
[24] J. Peise, B. Lücke, L. Pezzè, F. Deuretzbacher, W. Ertmer, J. Arlt, A. Smerzi, L. Santos, and C. Klempt, Nat. Commun. 6, 6811 (2015).

[25] X. Wang and B. C. Sanders, Phys. Rev. A 68, 033821 (2003).

[26] C. Gross, H. Strobel, E. Nicklas, T. Zibold, N. Bar-Gill, G. Kurizki, and M. K. Oberthaler, Nature (London) 480, 219 (2011).

[27] U. Leonhardt, Measuring the Quantum State of Light (Cambridge University Press, Cambridge, England, 1997), Chap. 5.

[28] A. I. Lvovsky and M. G. Raymer, Rev. Mod. Phys. 81, 299 (2009).

[29] C. Klempt, O. Topic, G. Gebreyesus, M. Scherer, T. Henninger, P. Hyllus, W. Ertmer, L. Santos, and J. J. Arlt, Phys. Rev. Lett. 103, 195302 (2009).

[30] L.-M. Duan, J. I. Cirac, and P. Zoller, Phys. Rev. A 65, 033619 (2002).

[31] M. Kitagawa and M. Ueda, Phys. Rev. A 47, 5138 (1993).

[32] T. Opatrný, Phys. Rev. A 91, 053826 (2015).

[33] M. Scully and M. Zubairy, Quantum Optics (Cambridge University Press, Cambridge, England, 1997).

[34] J. Peise, I. Kruse, K. Lange, B. Lücke, L. Pezzè, J. Arlt, W. Ertmer, K. Hammerer, L. Santos, A. Smerzi, and C. Klempt, Nat. Commun. 6, 8984 (2015).

[35] C. D. Hamley, C. S. Gerving, T. M. Hoang, E. M. Bookjans, and M. S. Chapman, Nat. Phys. 8, 305 (2012).

[36] D. Linnemann, H. Strobel, W. Muessel, J. Schulz, R. J. Lewis-Swan, K. V. Kheruntsyan, and M. K. Oberthaler, Phys. Rev. Lett. 117, 013001 (2016).

[37] M. Gabbrielli, L. Pezzè, and A. Smerzi, Phys. Rev. Lett. 115, 163002 (2015).

[38] G. Santarelli, P. Laurent, P. Lemonde, A. Clairon, A. G. Mann, S. Chang, A. N. Luiten, and C. Salomon, Phys. Rev. Lett. 82, 4619 (1999).

[39] J. Millo, M. Abgrall, M. Lours, E. M. L. English, H. Jiang, J. Guéna, A. Clairon, M. E. Tobar, S. Bize, Y. Le Coq, and G. Santarelli, Appl. Phys. Lett. 94, 141105 (2009).

[40] S. Weyers, B. Lipphardt, and H. Schnatz, Phys. Rev. A 79, 031803 (2009).

[41] G. Dobrev, V. Gerginov, and S. Weyers, Phys. Rev. A 93, 043423 (2016).

[42] H. Müntinga et al., Phys. Rev. Lett. 110, 093602 (2013).

[43] S. M. Dickerson, J. M. Hogan, A. Sugarbaker, D. M. S. Johnson, and M. A. Kasevich, Phys. Rev. Lett. 111, 083001 (2013).

[44] J. Rudolph, W. Herr, C. Grzeschik, T. Sternke, A. Grote, M. Popp, D. Becker, H. Müntinga, H. Ahlers, A. Peters, C. Lämmerzahl, K. Sengstock, N. Gaaloul, W. Ertmer, and E. M. Rasel, New J. Phys. 17, 065001 (2015).

[45] L. Pezzé and A. Smerzi, Phys. Rev. Lett. 100, 073601 (2008). 